\title{
Discussões sobre a Relação entre Limite e Continuidade de uma Função: investigando Imagens Conceituais
}

\author{
Discussions about the Relation between Limit and Continuity of a Function: \\ investigating Concept Images
}

\author{
Maria Alice de Vasconcelos Feio Messias ${ }^{*}$ \\ João Cláudio Brandemberg ${ }^{* *}$
}

\begin{abstract}
Resumo
Este trabalho apresenta resultados de uma investigação realizada junto a estudantes universitários que cursavam o $3^{\circ}$ e o $4^{\circ}$ semestre de licenciatura em matemática de duas universidades públicas do estado do Pará (Brasil). Objetivamos investigar as imagens conceituais desses sujeitos acerca da relação entre limite e continuidade de uma função, por meio de um estudo exploratório realizado em duas etapas, cujos resultados foram relacionados às pesquisas de Tall e Vinner (1981), Vinner (1991) que, por sua vez, compuseram a base da fundamentação teórica de nosso estudo. Dentre as imagens conceituais evocadas pelos sujeitos investigados, ressaltamos aquelas voltadas para a ideia de que o fato de uma função não estar definida em determinado ponto do domínio implica, necessariamente, na não existência do limite da função naquele ponto. Ou seja, de acordo com os sujeitos, a existência do limite depende da continuidade da função.
\end{abstract}

Palavras-chave: Imagem Conceitual. Limite de uma Função. Continuidade.

\begin{abstract}
This paper presents the results of an investigation that was made with university students enrolled in the $3^{\text {rd }}$ and $4^{\text {th }}$ semester of graduation in mathematics at two public universities in the state of Pará (Brazil). We aimed to investigate the concept image of these subjects about the relation between limit and continuity of a function, through an exploratory study done in two stages. The results were related to the researches of Tall and Vinner (1981), Vinner (1991) that composed our theoretical framework. Among the evoked concept images, we emphasize the idea that when a function is not defined in one point of the domain implies, necessarily, in the non-existence of the limit of that function in that point. That is, according to them, the limit's existence depends on the continuity of the function.
\end{abstract}

Keywords: Concept Image. Limit of a Function. Continuity.

\section{Introdução}

\footnotetext{
* Mestre em Educação em Ciências e Matemáticas pela Universidade Federal do Pará (UFPA). Docente no Departamento de Matemática da Universidade Federal do Maranhão (UFMA), São Luís, Maranhão, Brasil. Endereço para correspondência: Trav. Dos Periquitos, no 12, apto 104, CEP 65075-610, São Luís, Maranhão, Brasil._E-mail: alice.messias@gmail.com.

** Doutor em Educação pela Universidade Federal do rio Grande do Norte (UFRN). Docente no Instituto de Ciências Exatas e Naturais (ICEN) e Instituto de Educação Matemática e Científica (IEMCI) da Universidade Federal do Pará (UFPA), Belém, Pará, Brasil. Endereço para correspondência: Passagem Santa Rosa, 240, Guamá, CEP: 66075-530, Belém, Pará, Brasil. E-mail: brand@ufpa.br.
} 
Desde as últimas décadas do século $\mathrm{XX}$, diversos estudos e pesquisas relatam dificuldades de aprendizagem em matemática, desde as séries iniciais até o segmento superior de ensino. No último, observamos que essas dificuldades se manifestam de maneira expressiva em Cálculo. Por isso, optamos por investigar a problemática da apreensão do conceito de limite de uma função de uma variável real ${ }^{1}$, dada sua importância para o entendimento de outros conceitos. Enfatizamos - neste artigo - a relação entre limite e continuidade de uma função, bem como suas implicações nas evocações de imagens conceituais relacionadas a tais conceitos.

Os aspectos discutidos neste trabalho constituíram-se como parte dos resultados de uma pesquisa de mestrado, na qual objetivamos investigar as imagens conceituais de universitários acerca do conceito de limite de uma função. Para isso, aplicamos um questionário para estudantes universitários do curso de licenciatura em matemática de duas universidades públicas no estado do Pará.

Nesse primeiro momento, tivemos a oportunidade de - através dos registros escritos dos sujeitos investigados - verificar suas evocações relacionadas ao nosso objeto de estudo. A segunda etapa de nossa pesquisa consistiu na realização de entrevistas com alguns dos sujeitos investigados, a fim de descrever e analisar mais profundamente as imagens conceituais evocadas por eles na etapa anterior. Ressaltamos, no entanto, que o presente artigo apresenta trechos da entrevista realizada com apenas um desses sujeitos.

Um dos temas que se fez presente durante as entrevistas foi a relação entre a existência (ou não existência) do limite e a continuidade de funções. As discussões levantadas norteadas tanto em registros escritos obtidos por meio do questionário, quanto em estudos anteriores a nossa pesquisa com foco pautado na relação limite $x$ continuidade - foram analisadas atrelando nosso estudo às noções de imagem conceitual e definição conceitual (TALL; VINNER, 1981; VINNER, 1991).

Optamos por essa temática, devido às dificuldades atreladas à apreensão conceitual de limite de uma função. Baseamo-nos tanto em nossa experiência, enquanto docentes de Cálculo, quanto em estudos realizados nesse âmbito, donde identificamos que, para muitos estudantes:

[...] calcular o limite de uma função num ponto A, nos casos mais "interessantes", resumir-se-á em descobrir uma maneira de "substituir o A em $f(x)$ " sem que tal procedimento implique na emergência de irritantes quocientes com denominador nulo. Em outras palavras, a ideia de aplicar truques adequados de manipulação algébrica que permitirão "eliminar" tais inconveniências. A questão da existência do

\footnotetext{
${ }^{1}$ A função de uma variável real será tratada no decorrer desse artigo somente pelo termo função.
} 
limite, ou mesmo do que ele significa, ficarão ainda nebulosas [...] (OLIMPIO, 2007, p. 44).

Percebemos, ainda, que os entraves relativos à aprendizagem do conceito de limite estão ligados, principalmente, à dificuldade em correlacionar as noções intuitiva e formal desse conceito. Concordamos com Rodríguez (2009, p.97) no sentido de que, normalmente:

O docente explica que uma função $f$ tem limite $L$ quando $x$ tende a $a$ se a distância entre as imagens da função e $L$ pode ser arbitrariamente pequena, sendo os valores de $x$ muito próximos de $a$. Depois que se comunica oralmente esta frase, relativamente compreensível, escreve-se a definição com épsilon e delta (...) e poucos são os indivíduos que complementam o que fora escrito no quadro com as explicações dadas (tradução nossa).

Os fundamentos de nossa pesquisa estão atrelados às noções de imagem conceitual e definição conceitual de Tall e Vinner (1981) e Vinner (1991). Isso porque, as atividades propostas, bem como as discussões realizadas nas etapas de investigação de nossa pesquisa possibilitariam a ativação da cela ${ }^{2}$ da imagem conceitual dos sujeitos investigados, permitindo-nos analisar suas interpretações relacionadas ao conceito de limite de uma função (MESSIAS; BRANDEMBERG, 2014). No tópico subsequente apresentamos mais esclarecimentos acerca desse referencial.

\section{Sobre as contribuições de David Tall e Shlomo Vinner para o quadro teórico}

A Imagem conceitual relativa a um conceito é constituída por associações não verbais efetivadas em nossa mente quando em contato com o nome de determinado conceito. Sendo assim, estão incluídas representações visuais, figuras mentais, impressões e experiências que podem ser traduzidas em formas verbais por meio dessas associações (VINNER, 1991). Tall e Vinner (1981, p.152) destacam que:

Devemos utilizar o termo imagem conceitual para descrever a estrutura cognitiva total associada ao conceito, o que inclui todas as figuras mentais, propriedades e processos associados. É construída no decorrer dos anos através de experiências de todos os tipos, diferindo sempre que o indivíduo encontra novos estímulos e maturidade (tradução nossa).

Ressaltamos que as experiências vivenciadas pelo indivíduo são de extrema importância para a formação de sua imagem conceitual que, por sua vez, não é necessariamente coerente com a definição a que se relaciona, haja vista que pode apresentar propriedades ou interpretações contraditórias. Ainda assim, a formação de uma imagem conceitual - por meio do exercício de múltiplas representações de um conceito - permite que

\footnotetext{
${ }^{2}$ Brandemberg (2010) utiliza tal expressão com o intuito de relacioná-la com o formato de um escaninho que, por sua vez, caracteriza a composição do sistema cognitivo.
} 
o sujeito recupere suas impressões e experiências relacionadas a esse conceito e, quem sabe, garantir sua contextualização (MESSIAS, 2013; MESSIAS; BRANDEMBERG, 2014).

Assumimos, portanto, que "adquirir um conceito significa formar uma imagem conceitual sobre ele. Saber de cor a definição de um conceito não garante seu entendimento. Entender, assim supomos, significa apresentar uma imagem conceitual" (VINNER, 1991, p. 69, tradução nossa).

O termo definição conceitual está atrelado às definições, memorizadas ou autoconstruídas por um indivíduo. É toda forma em palavras utilizada para especificar um conceito, podendo sofrer variações à medida que o sujeito é submetido a novos estímulos (MESSIAS; BRANDEMBERG, 2014).

Para Cornu (1983, p.66), o termo definição conceitual consiste em um conjunto de palavras adquiridas em uma composição própria a cada indivíduo. Em suas palavras, define esse termo como:

[...] frases apreendidas mecanicamente, mais ou menos ligadas a um conceito; pode ser uma reconstrução, uma reformulação pessoal de uma definição matemática; é também o conjunto de palavras que empregamos para explicar o conceito. Essa fraseologia é própria ao indivíduo: ela não coincide sempre com a definição formal do conceito, ou seja, com a definição comumente admitida pela comunidade matemática (CORNU, 1983, p.66, tradução nossa).

Meyer (2003) destaca que imagem e definição conceitual pontuam a diferença entre um conceito matemático quando formalmente definido e os processos cognitivos pelo qual esse conceito é concebido. Sendo assim:

[...] uma pessoa pode criar uma ou múltiplas representações mentais para um mesmo conceito matemático, podemos inferir que é apoiado nessas representações que se torna possível a ampliação concreta do número de representações simbólicas ligadas a um determinado conceito, posto que cada representação mental estará associada a seu modelo de representação simbólica (BRANDEMBERG, 2010, p. 115).

Apresentamos, no tópico a seguir, ilustrações de imagens conceituais que se fizeram presentes em evocações de estudantes investigados em outras pesquisas sobre os conceitos de limite de função e continuidade. Baseados nessas pesquisas, destacamos a relação do suporte teórico sobre o conceito de limite com alguns questionamentos por nós levantados que, por sua vez, motivaram a construção de nosso instrumento de obtenção de dados e nortearam as discussões estabelecidas a partir das entrevistas.

\section{Exemplificando imagens conceituais sobre limite e continuidade por meio de pesquisas anteriores}


O estudo realizado por Cottrill et al. (1996) foi baseado na teoria $\operatorname{APOS}^{3}$ (Action Process Object Schema) e objetivou por em prática suas perspectivas teóricas, conhecimento matemático e análises acerca da aprendizagem de estudantes universitários no que concerne à noção de limite. Os dados obtidos na pesquisa foram levantados a partir da observação e das entrevistas realizadas com os estudantes que faziam parte de um curso experimental de Cálculo, tendo como estratégia pedagógica a combinação de atividades computacionais, tarefas no ambiente lápis e papel e exercícios.

As atividades propostas no decorrer da pesquisa se basearam em investigações computacionais de aproximação, investigações gráficas do conceito de limite, construções computacionais da aproximação de um valor ao limite, construções computacionais do conceito de limite e investigações $\operatorname{com} \varepsilon, \delta$. Dentre os dados obtidos na pesquisa, os autores observaram que:

(i) Para alguns estudantes o limite de uma função $f$ em um ponto $a$ não difere do valor da função $f$ no ponto $a$, ou seja, $\lim _{x \rightarrow a} f(x)=f(a)$.

(ii) Alguns sujeitos investigados apresentaram uma visão estática ${ }^{4}$ acerca do conceito de limite, na qual estabeleciam uma investigação da função em um único ponto e não em um grupo de valores.

(iii) O limite é uma aproximação dinâmica ${ }^{5}$ de $x$, entretanto, ao defini-lo atribuíam a noção de uma aproximação da função $f$ de um único valor de $x$ próximo de $a$.

Em (i), evidenciamos claramente que os indivíduos participantes da pesquisa de Cottrill et al (1996) mobilizaram, em suas imagens conceituais, a ideia de que a existência do limite em determinado ponto está condicionada à continuidade da função naquele ponto e que, portanto, $\lim _{x \rightarrow a} f(x)=f(a)$.

Barto (2004) objetivou investigar e analisar a produção de significados de estudantes de pós-graduação em educação matemática em relação à continuidade de funções de uma variável, buscando, dentre outras situações, verificar como ocorre a dinâmica da produção de significados para a continuidade e quais os significados produzidos para objetos matemáticos como ponto, curva, intervalo, épsilon, delta, domínio e imagem de funções.

\footnotetext{
${ }^{3}$ Baseada na teoria de Piaget; caracteriza a formação de determinado conceito matemático mediante um estágio em que é considerado um processo para outro, no qual passa a adquirir o status de objeto através de quatro estágios: Ação, Processo, Objeto e Esquema.

${ }^{4}$ Encontra-se atrelada à ideia de investigação do limite somente em um ponto, ou seja, a aproximação em torno do mesmo - que pode ou não estar definido no intervalo $(\mathrm{x}-\delta, \mathrm{x}+\delta)$ - não é considerada.

${ }^{5}$ São consideradas aproximações em torno de um ponto, a fim de calcular o valor do limite nesse ponto, dando a ideia de movimento; dinamismo.
} 
Barto (2004) observou os sujeitos participantes da disciplina Tópicos de Cálculo Diferencial e Integral e em seguida, os entrevistou, questionando-os sobre aspectos relacionados às tarefas realizadas em sala. Dentre os argumentos que emergiram durante a pesquisa da autora, evidenciamos que:

(iv) Para os sujeitos investigados, calcular limite de uma função significa realizar uma investigação à esquerda e à direita do ponto, independente desses valores serem iguais.

(v) Os estudantes calculavam os limites laterais, considerando-os satisfatórios para determinar se uma função era ou não contínua.

(vi) Função contínua é aquela que não tem saltos ou buracos.

Os itens (v) e (vi) mostram que o conceito de continuidade constituiu-se como um fator de conflito potencial para os sujeitos investigados por Barto (2004). Isso porque, em alguns momentos eles utilizavam o cálculo de limites laterais para determinar a continuidade de uma função. No entanto, quando os mesmos sujeitos investigavam representações gráficas de funções, concluíam que uma função contínua não apresenta saltos ou buracos que, por sua vez, não foram - em nenhum momento - relacionados com o fato de o limite não existir ou $\lim _{x \rightarrow a} f(x) \neq f(a)$.

Jordaan (2005) objetivou identificar os erros conceituais apresentados por estudantes de engenharia elétrica da Tshwane University of Technology (África do Sul) sobre o conceito de limite de função, no qual verificou como os sujeitos investigados entendiam a noção de limite, que tipo de erros apresentavam e como relacionavam (des) continuidade em um ponto à (não) existência do limite (MESSIAS; BRANDEMBERG, 2014). Os resultados obtidos por Jordaan (2005) permitiram que enunciasse as seguintes observações:

(vii) A função deve estar definida em um ponto para que a mesma apresente limite nesse ponto.

(viii) Para o limite existir em determinado ponto, a função deve ser contínua nesse ponto.

(ix) $\lim _{x \rightarrow a} f(x)=f(a)$, isto é, o limite é o valor da função no ponto.

Evidenciamos nos itens (vii), (viii) e (ix) que os indivíduos investigados na pesquisa de Jordaan (2005) também evocaram em suas imagens conceituais a ideia de que para o limite existir em determinado ponto do domínio, a função deve estar definida nesse ponto. Ou seja, para esses sujeitos, a existência do limite - em um primeiro momento - depende da continuidade da função. 
Em sua pesquisa, Nair (2010) investigou imagens conceituais de estudantes de Cálculo relativas aos conceitos de função racional, assíntotas, limites e continuidade. A autora objetivou verificar que conexões entre os conceitos de assíntotas, continuidade e limite de funções racionais os sujeitos investigados apresentavam. Sua pesquisa constituiu-se de uma entrevista, na qual as imagens conceituais identificadas subsidiaram a elaboração de planos de aula que foram usados nos episódios de ensino realizados ao longo da investigação (MESSIAS; BRANDEMBERG, 2014).

No que se refere à relação entre os conceitos de limite e continuidade, Nair (2010) assim como Cottril et al (1996) e Jordaan (2005) - evidenciou que:

(x) Os sujeitos investigados acreditavam que o limite não existe em determinado ponto se a função não estiver definida naquele ponto.

(xi) Os estudantes acreditam que o $\lim _{x \rightarrow a} f(x)$ deve ser necessariamente $f(a)$. Caso contrário, o limite não existirá.

Os resultados obtidos nesses estudos fomentaram a elaboração de questionamentos que, por sua vez, permitiram-nos estruturar, tanto o questionário aplicado na $1^{\text {a }}$ etapa de nossa investigação, quanto o roteiro que norteou as discussões estabelecidas durante as entrevistas com os sujeitos investigados. Destacamos, no quadro 1, as implicações das imagens conceituais identificadas nos referenciais teóricos adotados para a formulação de nossas questões de pesquisa.

\begin{tabular}{|c|c|c|}
\hline Referencial Teórico & Imagens Conceituais Evocadas & Questões norteadoras em nosso estudo \\
\hline Cottrill et al (1996) & $\lim _{x \rightarrow a} f(x)=f(a)$ & \multirow{4}{*}{$\begin{array}{l}\text { 1. Como os sujeitos investigados em nossa } \\
\text { pesquisa relacionam os conceitos de limite e } \\
\text { continuidade? } \\
\text { 2. Os sujeitos investigados condicionam a } \\
\text { existência do limite em determinado ponto à } \\
\text { continuidade da função nesse ponto? } \\
\text { 3. A definição conceitual pessoal dos } \\
\text { sujeitos investigados acerca do conceito de } \\
\text { limite evoca a noção de continuidade? } \\
\text { 4. O que os sujeitos investigados em nossa } \\
\text { pesquisa consideram como necessário para } \\
\text { que um limite exista em torno de um } \\
\text { determinado ponto? } \\
\text { 5. Como os sujeitos investigados visualizam } \\
\text { a (des) continuidade de uma função em um } \\
\text { ponto e, também, a existência (ou não } \\
\text { existência) do limite nesse ponto por meio } \\
\text { de representações gráficas? }\end{array}$} \\
\hline Barto (2004) & $\begin{array}{l}\text { Verifica-se a continuidade de uma } \\
\text { função em um ponto, por meio do } \\
\text { cálculo dos limites laterais, à direita e à } \\
\text { esquerda desse ponto. } \\
\text { Uma função é contínua se não } \\
\text { apresenta saltos ou buracos. }\end{array}$ & \\
\hline Jordaan (2005) & $\begin{array}{l}\text { A função deve estar definida em um } \\
\text { ponto para que a mesma apresente } \\
\text { limite naquele ponto. } \\
\text { Quando a função tem um limite em um } \\
\text { ponto, então deve ser contínua naquele } \\
\text { ponto; } \lim _{x \rightarrow a} f(x)=f(a)\end{array}$ & \\
\hline Nair (2010) & $\lim _{x \rightarrow a} f(x)=f(a)$ & \\
\hline
\end{tabular}

Quadro 1 - Implicações do referencial teórico para a formulação de nossas questões de pesquisa 
Tais questões norteadoras em nosso estudo propiciaram algumas reflexões acerca de como poderíamos estruturar os momentos de investigação junto aos sujeitos investigados, de maneira a ser possível identificar se os mesmos evocariam imagens conceituais semelhantes àquelas destacadas em nosso quadro de referências.

\section{Algumas reflexões relacionadas à estrutura e outros os aspectos de nossa investigação}

Em nossa pesquisa, objetivamos investigar - através de um questionário e sessões de entrevistas - as imagens conceituais evocadas ao resolverem questões envolvendo o conceito de limite de função. E, como identificamos em pesquisas anteriores que a relação limite $x$ continuidade é um fator de conflito potencial para muitos estudantes, optamos por explorar, junto aos sujeitos investigados, essa relação e as implicações que ela pode ter na formação de suas imagens conceituais no que se refere ao conceito de limite de função.

Ressaltamos que este trabalho apresenta parte dos resultados de uma pesquisa de mestrado que foi realizada em duas etapas. Na primeira, os sujeitos responderam a um questionário e, na segunda, selecionamos alguns sujeitos para serem entrevistados. Para isso, elaboramos um roteiro específico para cada estudante, levando em consideração as imagens conceituais sobre o conceito de limite de uma função, evocadas na etapa anterior. Para responder às questões de pesquisa concernentes à relação limite $x$ continuidade, solicitamos aos sujeitos investigados que respondessem a seguinte questão (Figura 1):

Observe os gráficos abaixo e responda:

1.1.

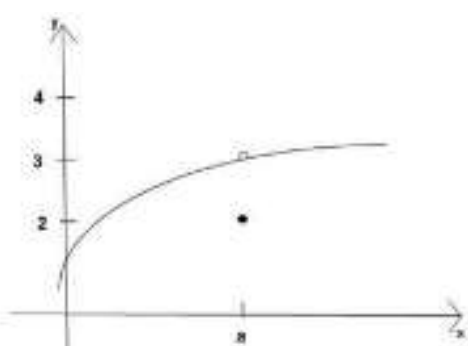

1.1) O limite da função em (1.1) existe em $\mathrm{x}=\mathrm{a}$ ? Justifique sua resposta
1.2 .

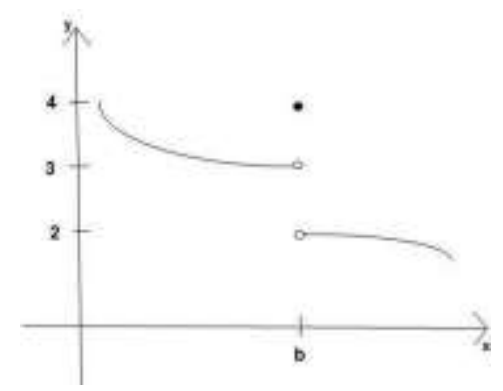

1.2) O limite da função em (1.2) existe em $x=b$ ? Justifique sua resposta

Figura 1 - A relação limite $x$ continuidade no questionário aplicado. Fonte: Messias (2013)

A elaboração dessa questão norteou-se nas considerações de Juter (2006) sobre as mobilizações das Imagens Conceituais de estudantes em relação ao comportamento da função próximo e no próprio valor do limite. Nosso intuito foi verificar como os estudantes 
interpretariam a (não) existência do limite em funções descontínuas. Nossas hipóteses, quanto aos resultados obtidos em nossa investigação, pautaram-se nas seguintes evocações:

- Para os sujeitos investigados, $\lim _{x \rightarrow a} f(x)=f(a)$ (COTTRILL et al., 1996).

- Calcular o limite de uma função significa realizar uma investigação à direita e à esquerda de um ponto qualquer (BARTO, 2004).

- Uma função que não esteja definida em um ponto não apresenta limite quando a função tende àquele ponto (JORDAAN, 2005).

- $\mathrm{O} \lim _{x \rightarrow a} f(x)$ deve ser necessariamente igual a $f(a)$. Caso contrário, o limite não existirá (NAIR, 2010).

\section{Uma análise acerca dos resultados obtidos na $1^{\text {a }}$ etapa}

$\mathrm{Na}$ primeira etapa de nossa investigação, contamos com a participação de 25 estudantes universitários que cursavam o $3^{\circ}$ e $4^{\circ}$ semestre do curso de licenciatura em matemática de duas universidades públicas no estado do Pará (Brasil), que já haviam cursado a disciplina Cálculo $I$. No caso da questão destacada na figura 1 deste artigo, objetivamos verificar como os estudantes interpretariam a (não) existência do limite em funções descontínuas, bem como a relação entre os limites laterais, a fim de observar a unicidade do limite. Observamos que alguns dos sujeitos investigados apresentaram dificuldade em interpretar os gráficos, isso porque, 7 dos 25 estudantes que responderam ao questionário não responderam a essa questão.

A investigação da aproximação à direita e à esquerda do ponto dado fez parte da resolução de 9 estudantes que atribuíram à existência do limite, o fato de seus limites laterais existirem e serem iguais (Figuras 2 e 3 ).

a) Existe, pois, conforme nos aproximamos de a, o valor de $f(x)$ aproxima-se do $\lim _{x \rightarrow a} f(x)$.

b) Não existe, pois quando nos aproximamos de a pela esquerda e pela direita obtemos valores diferentes para $\lim _{x \rightarrow a} f(x)$. Logo, $\lim _{x \rightarrow a} f(x)$ não existe.

Figura 2 - Transcrição da resposta do Sujeito S12

Fonte: Messias (2013) 
5) a.1) Sim.

a.2) Quando calculamos os limites laterais da função, com x tendendo a qualquer valor, eles serão iguais. Quando $x$ tender à a, teremos $\lim _{x \rightarrow a^{+}} f(x)=\lim _{x \rightarrow a^{-}} f(x)$ (analisando o gráfico).

b.1) Existirá em todos os pontos, exceto em $x=b$.

b.2) Quando calcularmos os limites laterais da função em qualquer ponto diferente de b, eles serão iguais (analisando o gráfico), mas quando x tender a $b$, os limites laterais da função serão diferentes $\left(\lim _{x \rightarrow b^{+}} f(x) \neq \lim _{x \rightarrow b^{-}} f(x)\right.$. Logo, a função não terá limite quando $x$ tender $a b$.

Figura 3 - Transcrição da resposta do Sujeito S14 Fonte: Messias (2013)

Nesse sentido, nossos resultados não estão em conformidade com os de Barto (2004), cujos sujeitos investigados consideravam somente as investigações à direita e à esquerda do ponto satisfatórias para a existência do limite, descartando a sua unicidade. De qualquer maneira, ainda assim observamos, nas respostas dos sujeitos, a mobilização de uma ideia de limite de função como sendo uma aproximação contínua em relação a um ponto sem, no entanto, atingir esse ponto (TALL; VINNER, 1981).

Evidenciamos em nossos resultados que - semelhante aos estudos de Cottrill et al. (1996), Jordaan (2005) e Nair (2010) - os sujeitos investigados condicionam a existência do limite de uma função à continuidade dessa função em todos os pontos do domínio, pois para "uma função satisfazer o conceito de limite...precisa ser contínua em todo o seu domínio" (resposta do sujeito S15). Nesse sentido, observamos que os sujeitos investigados apresentam mobilizações em suas imagens conceituais semelhantes às de Leibniz no séc. XVII, em que a existência do limite dependia da continuidade da função no ponto e não o contrário.

Verificamos que alguns sujeitos evocaram, em suas imagens conceituais, que a continuidade de uma função está condicionada a não existência de saltos no gráfico da função (TALL; VINNER, 1981; BARTO, 2004), conforme elucidamos na resposta do sujeito S20.

5) A função g tem limite pois não possui salto em a, porém não é contínua em a.

b) A função h não possui limite pois apresenta salto em $b$.

Figura 4 - Transcrição da resposta do Sujeito S20

Fonte: Messias (2013)

Somente a resposta do sujeito S09 demonstrou estar de acordo com as considerações de Barto (2004), dado que se preocupou somente com as investigações à direita e à esquerda do ponto dado. Além disso, observamos para o referido sujeito, os saltos no gráfico implicam na existência de duas funções diferentes. Portanto, ao contrário da maioria dos participantes 
de nossa pesquisa, ele não evocou a ideia de continuidade para responder à questão, conforme evidenciamos em sua resposta:

5) a) Sim. Pois, este gráfico mostra claramente o comportamento de x quando tende ao seu limite, que neste caso tem-se o ponto a como tendência admitindo um único valor.

b) Sim, porém, não é única função, nesta existem 2, porém, com um valor único como tendência.

Figura 5 - Transcrição da resposta do Sujeito S09

Fonte: Messias (2013)

Elucidamos que, a partir das análises dos resultados obtidos, identificamos que $\mathrm{s}$ seguintes imagens conceituais foram evocadas, no que concerne à relação limite $x$ continuidade:

- Para o limite de uma função existir, seus limites laterais devem ser iguais.

- O conceito de limite de função encontra-se relacionado com uma investigação nas proximidades de determinado ponto, de maneira que a função se aproxime de L sem, no entanto, alcançar esse limite. Ou seja, $\boldsymbol{f}(\boldsymbol{x}) \neq \boldsymbol{L}$ (TALL\&VINNER, 1981; JUTER, 2006) .

- Para que o limite de uma função exista, a função deve estar definida no ponto, ou seja, deve ser contínua nesse ponto (COTTRILL et al, 1996; JORDAAN, 2005; NAIR, 2010) .

- O limite de uma função existe se não houver saltos no gráfico da função.

A análise das imagens conceituais evocadas pelos sujeitos subsidiou a elaboração do roteiro utilizado durante as entrevistas. Optamos, nesse sentido, por investigar mais profundamente a relação limite $x$ continuidade, por meio do Tema de Discussão (TD) intitulado: (Des) continuidade implica na (não) existência do limite?. O objetivo desse TD voltou-se para a análise das imagens conceituais evocadas, conforme os resultados obtidos por Cottrill et al. (1996), Jordaan (2005) e Nair (2010), ou seja, conforme a percepção de que para o limite de uma função existir em determinado ponto, a função deve ser contínua nesse ponto.

\section{6 (Des) continuidade implica na (não) existência do limite?}

Com o intuito de complementar os resultados obtidos no que concerne à relação limite $x$ continuidade, elaboramos um roteiro de entrevista para discutir junto aos sujeitos 
investigados que mobilizaram em suas imagens conceituais os aspectos descritos no quadro 1 . Nossas discussões nortearam-se, como segue:

Mostrar o gráfico da função abaixo:

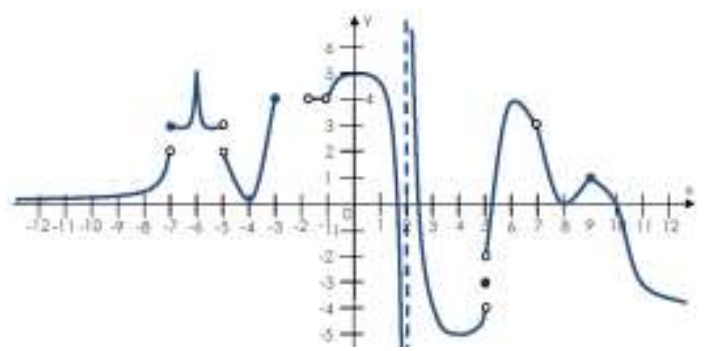

- $\quad$ Observe esse gráfico e responda:

$\circ \quad \mathrm{O} \lim _{x \rightarrow-7} f(x)$ existe? Justifique.

- E quando $x \rightarrow 5$ ? Justifique.

○ $\mathrm{O} \lim _{x \rightarrow 7} f(x)$ existe? Justifique.

○ E quando $x \rightarrow 9$ ? Justifique.

- Escreva uma definição para $\lim _{x \rightarrow a} f(x)=L$ e, em seguida, explique-a.

- Caso seja mobilizada a ideia de que o limite existe se $\lim _{x \rightarrow a} f(x)=f(a)$, perguntar:

- Então, o limite da função em determinado ponto deve ser igual ao valor da função nesse ponto? (aguardar resposta). E se não for?

○ Devemos, portanto, considerar o domínio da função como um fator decisivo para evidenciarmos a (não) existência do limite?

○ Então quando o limite de uma função existe?

- Escreva uma definição para $\lim _{x \rightarrow a} f(x)=L$ e, em seguida, explique-a.

- Caso o sujeito responda corretamente o primeiro tópico, solicitar que responda:

○ Quando o limite existe?

Figura 6 - A relação limite $x$ continuidade explorada nas sessões de entrevista Fonte: Messias (2013)

O roteiro de entrevista (figura 6) explora a relação entre (des) continuidade de função em um ponto e a (não) existência do limite nesse ponto. Incluímos um gráfico de função descontínua para ser analisado pelos sujeitos. Nosso intuito foi levantar possíveis evocações que estivessem voltadas para a ideia de que o limite de uma função existe se $\lim _{x \rightarrow a} f(x)=f(a)$. Acreditamos, também, ser importante pedir que os sujeitos definissem limite de função, bem como explicassem o seu significado, pois dessa maneira poderíamos comparar as imagens conceituais evocadas e definição conceitual pessoal com a definição conceitual formal de limite de função. Mais uma vez, ressaltamos que o referido roteiro norteou-se, principalmente, nos estudos de Cottrill et al. (1996), Jordaan (2005) e Nair (2010).

\section{Uma análise acerca das discussões estabelecidas com os sujeitos investigados}


A escolha do sujeito investigado deu-se a partir da análise dos resultados obtidos na etapa anterior. Ou seja, selecionamos um estudante, cujas imagens conceituais evocadas melhor representassem o TD em questão. Nesse sentido, solicitamos a participação do sujeito S09 e exploramos junto a ele suas percepções voltadas para a relação entre limite $e$ continuidade. Ressaltamos que S09 evocou, na etapa anterior, imagens conceituais que contemplaram a ideia de que para o limite existir a função deve ser contínua, ou seja, $\lim _{x \rightarrow a} f(x)=f(a)$.

A investigação foi realizada na última semana de setembro de 2012, e a entrevista durou em torno de 15 minutos. A análise das entrevistas foi fundamental para complementar nossas interpretações acerca das imagens conceituais evocadas pelos sujeitos investigados na $1^{a}$ etapa. Destacamos, neste trabalho, a descrição de alguns trechos referentes às discussões estabelecidas, analisando-os de acordo com os pontos de conformidade e/ou não conformidade com o referencial teórico previamente estabelecido. Apesar de termos elaborado um roteiro para o TD, realizamos outros questionamentos que pudessem complementar nossas análises, sendo esses questionamentos realizados conforme as particularidades do sujeito investigado.

No decorrer da entrevista, o sujeito S09 evocou que a existência do limite em determinado ponto não está, necessariamente, atrelada à (des) continuidade, conforme elucidamos a seguir (Figura 7):

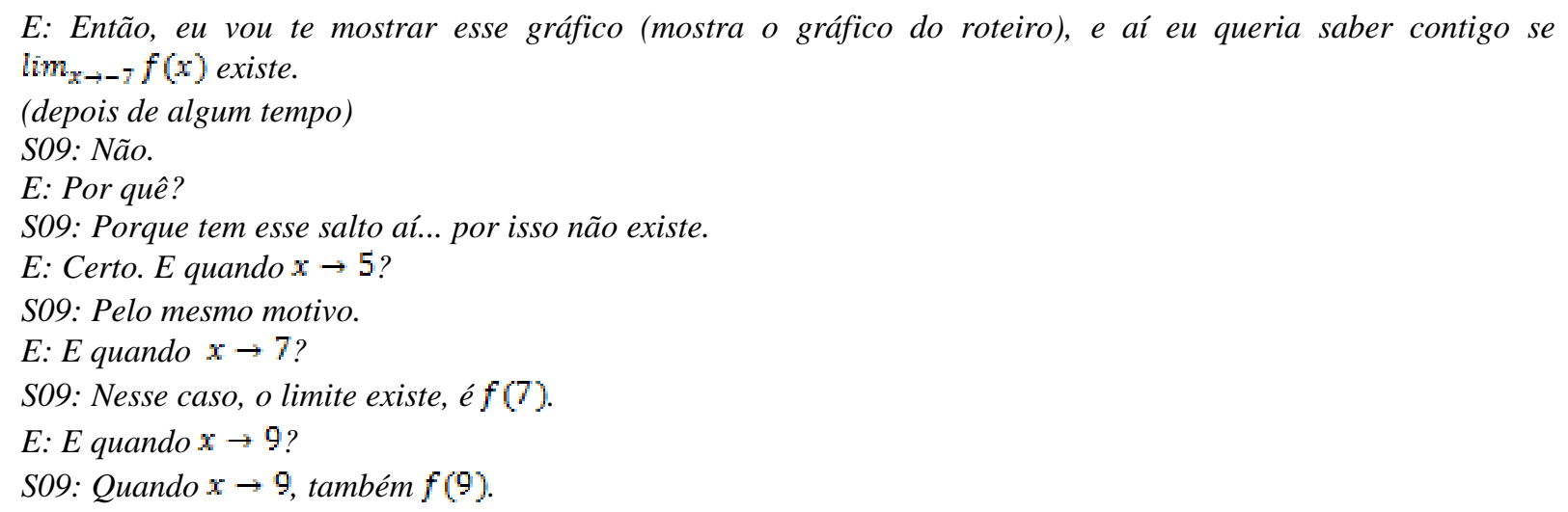

Figura 7 - Trecho 1: Entrevista sujeito S09 Fonte: Messias (2013)

Nos pontos $x=-7$ e $x=5$, o limite - conforme destacado por S09 - não existe, sendo que esse salto elucidado pelo sujeito é uma consequência dos limites laterais serem diferentes (no caso de $X=-7, \lim _{x \rightarrow-7^{+}} f(x)=3$ e $\lim _{x \rightarrow-7^{-}} f(x)=2$ ). Entretanto, em nenhum momento S09 mencionou que o fato dos limites laterais serem diferentes implica na 
não existência do limite no ponto. Para ele, o limite não existe em virtude de a função ter um salto $^{6}$. Nesse sentido, nossos resultados se assemelham aos de Tall e Vinner (1981).

A transcrição não nos permite afirmar que para S09 a existência do limite em determinado ponto dependa da continuidade nesse ponto, haja vista que em $x=7$ a função é descontínua. Todavia, segundo ele, o limite existe e seria igual a $f(7)$. Entretanto, evidenciamos que, de alguma maneira, a relação (des)continuidade x (não)existência do limite faz parte de sua imagem conceitual. Pautamos essa afirmação nas seguintes possibilidades:

- O sujeito atrela à ideia de descontinuidade somente a existência dos saltos no gráfico de uma função. Se for esse o caso, então, para o sujeito, a existência do limite dependeria da continuidade da função (no caso de $\lim _{x \rightarrow-7} f(x)$, por exemplo), fato que aproximaria nossos resultados com os Cottrill et al. (1996), Jordaan (2005) e Nair (2010) .

- Para o sujeito, uma função é descontínua quando a mesma apresenta saltos e buracos em um ponto $P$ (BARTO, 2004). Sendo que, nesse último caso, o limite existirá e assumirá o valor de $f(P)$, alcançando-o, ou seja, ele se aproximará tanto de $f(p)$ que se tornará esse valor (TALL; VINNER, 1981).

No decorrer da entrevista, observamos que o sujeito menciona os intervalos na tentativa de explicar quando o limite em determinado ponto existe ou não existe, sendo que o domínio da função se configurou como um fator de conflito potencial (VINNER, 1991) para esse sujeito, conforme destacamos na transcrição a seguir (Figura 8):

E: Então, quando eu posso afirmar que o limite existe?

S09: Vamos dizer que eu quero calcular o limite de $f$ quando $x \rightarrow a$, para pontos cada vez mais próximos de a no domínio, o valor das imagens tem que tá cada vez mais próxima de $f(a)$.

E: Então, eu devo considerar o domínio da função como sendo fundamental para a existência ou não existência do limite?

S09: Eu acho que não o domínio e sim as imagens.

E: então o valor da função no ponto deve pertencer ao conjunto imagem para que o limite exista?

S09: É.

E: Então, quando o limite não existe?

S09: Seria (pausa) se tu determinas um intervalo próximo de a e um intervalo próximo de $f(a)$. (depois de algum tempo). Tá, o limite não existe se eu tomar um intervalo próximo de a, contendo a, eu pegar algum valor desse intervalo e a imagem não pertencer ao intervalo próximo de $f(a)$.

E: Então o caso do intervalo em torno de a, a tem que estar definido nesse intervalo?

S09: tem que tá no intervalo.

Figura 8 - Trecho 2: Entrevista sujeito S09

Fonte: Messias (2013)

\footnotetext{
${ }^{6} \mathrm{O}$ sujeito interpreta o salto no gráfico de uma função como uma característica de descontinuidade que, por sua vez, implicaria - segundo sua imagem conceitual evocada - na não existência do limite dessa função.
} 
Diante das respostas de S09, percebemos, também, que o mesmo considera o limite como sendo uma aproximação dinâmica em relação à $x$, conforme evidenciamos em suas palavras: "para pontos cada vez mais próximos de $a$...”. Entretanto, quando define o valor do limite em $P$, restringe-se a esse ponto, já que no caso do salto em $x=-7$, não analisou o comportamento do gráfico nas proximidades desse ponto e, consequentemente, desconsiderou seus limites laterais. Nesse âmbito, nossos resultados assemelham-se com as considerações de Cottrill et al. (1996).

Ressaltamos, ainda, que o sujeito S09 mostrou-se inseguro quanto ao questionamento levantado acerca da necessidade de a função estar ou não definida no ponto para que o limite exista. Em um primeiro momento, diz que o domínio da função não deve ser considerado e, sim, a imagem. No entanto, afirma que o ponto $a$ deve estar definido no intervalo. Acreditamos, nesse aspecto, que os próprios conceitos de domínio e imagem mostraram-se como fatores de conflito potencial.

\section{Considerações finais}

Com essa pesquisa nos propusemos a investigar os elementos que compõem a imagem conceitual de estudantes universitários sobre limite de função, inferidos a partir da evocação de aspectos relacionados a esse conceito. Os resultados obtidos nos subsidiaram a verificar as imagens conceituais evocadas pelos estudantes investigados no que concerne ao conceito de limite de função, bem como sua relação com a noção de continuidade. Nesse sentido, observamos que as imagens conceituais dos sujeitos pautaram-se, sobretudo, nas duas importantes evocações a seguir:

[E1] A ideia de limite como sendo um valor a ser alcançado pela função por meio de constantes aproximações. Desse modo, o limite em determinado ponto é tido como um valor que deve coincidir com o valor da função nesse ponto, ou seja, $\lim _{x \rightarrow p} f(x)=f(p)$.

[E2] A existência do limite em um ponto $p$ depende da continuidade em $p$.

Ressaltamos que a evocação E1 assemelha-se aos resultados obtidos por Tall e Vinner (1981), Jordaan (2005) e Juter (2006). Isso porque, nossos resultados permitiram-nos evidenciar que, de fato, os estudantes pesquisados relacionavam o conceito de limite de função com interpretações estáticas e/ou dinâmicas que, em alguns momentos, constituíramse como fatores de conflito potencial (VINNER, 1991), principalmente no que concerne à 
possibilidade do valor do limite poder ou não ser alcançado (MESSIAS; BRANDEMBERG, 2014).

E2 encontra-se em conformidade com as pesquisas realizadas por Cottrill et al. (1996), Jordaan (2005) e Nair (2010). As evocações E1 e E2 nos permitiram evidenciar que algumas das imagens conceituais evocadas pelos sujeitos investigados não se fizeram coerentes com a definição conceitual, fato que os influenciou a construir uma definição conceitual pessoal diferente da definição conceitual formal de limite de função.

Evidenciamos - por meio das imagens conceituais evocadas pelos sujeitos investigados - os elementos que compõem essas mobilizações. Além disso, observamos que existem divergências entre a definição conceitual pessoal apresentada pelos estudantes e a sua definição conceitual formal. Sendo assim, acreditamos que as duas etapas da pesquisa nos possibilitaram responder aos nossos questionamentos e alcançar, de maneira satisfatória, os objetivos traçados para esta pesquisa, uma vez que conseguimos identificar as principais dificuldades relacionadas à apreensão do conceito de limite de uma função, correlacionar imagens conceituais evocadas, definição conceitual pessoal e definição conceitual formal desse objeto de estudo e, por fim, elucidar os pontos de conformidade e/ou não conformidade desses resultados em relação à fundamentação teórica preestabelecida.

Consideramos os resultados obtidos em nossa investigação relevantes no sentido de que permitem verificar alguns conflitos que permeiam a construção de imagens conceituais dos estudantes em relação ao conceito de limite. Nossos resultados, em um primeiro momento, subsidiaram discussões realizadas pelos membros do Grupo de Estudos e Pesquisa em História e Ensino da Matemática - GEHEM ${ }^{7}$. Durante as reuniões, alguns apontamentos foram realizados, tais como a possibilidade de usar a História da Matemática como componente metodológica para o ensino de conteúdos de Cálculo, inclusive os conceitos de limite e continuidade de uma função.

Nesse âmbito, em Messias e Brandemberg (2013), apontamos que atividades contemplando episódios da história do desenvolvimento do Cálculo podem ser utilizadas para auxiliar no entendimento dos conceitos de limite e continuidade de uma função. Tais episódios podem ser relacionados, por exemplo, a dois marcos importantes da história do Cálculo: os paradoxos de Zenão de Elea e a Quadratura da Parábola.

\footnotetext{
${ }^{7}$ Vinculado ao Instituto de Educação Matemática e Científica (IEMCI) da Universidade Federal de Pará (UFPA); fundado em 2012 e coordenado por professores credenciados ao Programa de Pós-Graduação em Educação em Ciências e Matemáticas da referida instituição.
} 
No caso dos paradoxos de Zenão, é possível permitir que os estudantes vivenciem as dúvidas relacionadas à ideia de movimento (de maneira semelhante aos gregos na antiguidade). Sob essa perspectiva, os estudantes podem buscar - intuitivamente - conceitos como o de continuidade, infinito e convergência que são necessários para solucionar tais paradoxos e, extrínsecos ao pensamento matemático grego da época. O método de exaustão, utilizado para encontrar a área do segmento parabólico, envolve a ideia de quantidades infinitamente pequenas que jamais chegam a zero e que caracterizam a área remanescente entre o polígono e a parábola. Esse episódio pode desafiar o comportamento dos estudantes no que concerne ao cálculo dessa área remanescente, bem como sua relação com o conceito de limite.

Ressaltamos que este estudo tem sido discutido pelos membros do GEHEM; a elaboração de uma sequência didática envolvendo a construção histórica dos conceitos de limite e continuidade encontra-se em andamento, e se configura como parte de um estudo maior, no qual estão incluídas pesquisas relacionadas ao ensino de outros conceitos - tais como os de derivada e integral - por meio da utilização da história da matemática como componente metodológica, de maneira a promover um ensino-aprendizagem de Cálculo que busque dar uma ressignificação aos conhecimentos matemáticos produzidos ao longo de seu desenvolvimento e, consequentemente, formar imagens conceituais coerentes com suas definições formais.

\section{Referências}

BARTO, M. C. Um olhar sobre as ideias matemáticas em um curso de Cálculo: a produção de significados para continuidade. 2004. 133f. Dissertação (Mestrado em Educação Matemática)Faculdade de Matemática, Pontifícia Universidade Católica, São Paulo, 2004.

BRANDEMBERG, J. C. Uma análise histórico-epistemológica do conceito de grupo. São Paulo: Livraria da Física (ED), 2010.

COTTRILL et al. Understanding the limit concept: beginning with a coordinate process scheme. Journal of mathematical behavior, New Jersey, v. 15, n.2, p. 167 - 192, jun. 1996.

CORNU, B. Apprentissage de la notion de limite - conceptions et obstacles. 1983. 169f. Tese (Doutorado em Matemática) - Institut de recherche sur l'enseignement dês mathématiques, Université Scientifique et Medicale de Grenoble, Grenoble, 1983.

JORDAAN, T. Misconceptions of the limit concept in a mathematics course for engineering students.2005. 87f. Dissertação (Mestrado em Educação) - University of South Africa, White River, 2005. 
JUTER, K. Limits of functions - University students' concept development. 2006. 175f. Tese (Doutorado em Educação Matemática). - Departamento de Matemática, Lulea University of Technology, Lulea, 2006.

MESSIAS, M.A.V.F; BRANDEMBERG, J.C. Selecionando episódios da história do cálculo para o ensino do conceito moderno de limite de uma função. In: SEMINÁRIO NACIONAL DE HISTÓRIA DA MATEMÁTICA, 10, 2013, Campinas, SP. Anais... São Paulo: Unicamp, 2013, p. 1 - 8, CDROM.

MESSIAS, M. A. V. F; BRANDEMBERG, J.C. Um estudo exploratório sobre as evocações de estudantes universitários acerca do conceito de limite de função. REVEMAT, Florianópolis (SC), v. 9, n.1, p.191 - 209, ago. 2014.

MESSIAS, M. A. V. F. Um estudo exploratório sobre a imagem conceitual de estudantes universitários acerca do conceito de limite de função. 2013. 133f. Dissertação (Mestrado em Educação em Ciências e Matemáticas) - Instituto de Educação Matemática e Científica, Universidade Federal do Pará, Belém, 2013.

MEYER, C. Derivada/Reta tangente: Imagem conceitual e Definição conceitual. 2003. 161f. Dissertação (Mestrado em Educação Matemática) - Faculdade de Matemática, Pontifícia Universidade Católica, São Paulo, 2003.

NAIR, G. S. College students' concept image of asymptotes, limits and continuity of rational functions. 2010. 276f. Tese (Doutorado em Filosofia) - College of Education and Human Ecology, Ohio State University, 2010.

OLIMPIO, A. Primeiro ano num curso de matemática: a definição de função e a dualidade local/global em conceitos de cálculo. Bolema, Rio Claro (SP), v. 20, n. 28, p. 39-67, jul. 2007.

RODRÍGUEZ, M. Consideraciones didácticas para la enseñanza del límite funcional. In: Simposio de Educación Matemática, 10, 2009, Chivilcoy. Memorias del $\mathbf{1 0}^{\circ}$ Simposio de Educación Matemática. Buenos Aires: Universidad Nacional de Lujan, 2009, 92 - 98. CD-ROM

TALL, D; VINNER, S. Concept image and concept definition with particular reference to limits and continuity. Educational Studies in Mathematics, New York, n. 12, p. 151 - 169, may. 1981.

VINNER, S. The role of definitions in the teaching and learning of mathematics. In: TALL, D (Ed.). Advanced Mathematical Thinking. Dordretch: Kluwer publications, 1991, p. 65 - 81 .

Submetido em Abril de 2015. Aprovado em Agosto de 2015. 